\title{
A MIXED DIRICHLET-NEUMANN PROBLEM FOR A NONLINEAR REYNOLDS EQUATION IN ELASTOHYDRODYNAMIC PIEZOVISCOUS LUBRICATION
}

\author{
by J. DURANY, G. GARCÍA and C. VÁZQUEZ
}

(Received 14th July 1994)

\begin{abstract}
The aim of this work is to study the existence of solutions for a mathematical model of the displacement of a piezoviscous lubricant between two elastic surfaces. As we deal with a rolling ball contact problem, the deformations are modelled by the linear Hertzian theory. The fluid pressure behaviour is governed by the classical Reynolds equation for thin film displacement. The relevant aspect of cavitation in lubrication is described by means of the Elrod Adams model which leads to a mathematical free boundary problem.

The two main original features of the model problem in relation to previous works are: the supply of lubricant coming from a groove that is transversal to the direction of fluid displacement and the consideration of a piezoviscous law of Barus. Mathematically, the first one leads to a mixed Dirichlet-Neumann problem for the Reynolds equation and the second one involves an additional nonlinearity in a diffusion type term.
\end{abstract}

1991 Mathematics subject classification: 35J25, 35J60, 35R35, 73T05

\section{Introduction}

The greatly increasing number of industrial technical devices involving the presence of lubricated contacts motivates interest in studying more suitable mathematical models for the different practical situations. The two elastic solids in contact can present several geometries: cylinder-cylinder, cylinder-plane and sphere-plane contacts, for example. A complete rigorous mathematical study might include not only the description of the numerical algorithms that provide results agreeing with experimental measures but also the theoretical proofs, based on tools of mathematical analysis, that ensure their validity.

We consider as solids both an elastic rolling ball and a rigid plane. The real possibility of the ball deformation leads to an elastohydrodynamic lubrication problem. Basic aspects of the early developed theory in this scientific domain can be found in Dowson-Higginson [8] where the three main common features of this kind of problem are already quoted: the fluid hydrodynamic displacement, the solid elastic deformation and the air bubble generation. The Reynolds equation, linear Hertz contact theory and different cavitation models try to mathematically model these three phenomena respectively. Moreover, the modification of the initial fluid viscosity due to the presence of sufficiently high values of lubricant pressure might have to be taken into account in certain devices. For this purpose, in this paper, we consider the piezoviscous relation given by Barus law (see Cameron [6]). 
In some cases, the set of equations that takes into account the different phenomena consists of a non-linear elliptic problem. In others, the behaviour of fluid pressure is modelled in terms of a variational inequality. For the latter, in the constant viscosity case, the works of Oden-Wu [13] and $\mathrm{Hu}$ [11] treat the problem in one and two dimensions. For this variational inequality cavitation model, existence results appear in $\mathrm{Hu}$ [11] and in Rodrigues [14] by using different ' $a$ priori' estimates. Nevertheless, as is pointed out in Bayada-Chambat ([1] and [2]), this model is not always appropriate to describe the cavitation phenomenon. An alternative model introduces a saturation function as a supplementary unknown, see Bayada-Chambat [1]. Consideration of this model also leads to the existence and uniqueness of the solution for a hydrodynamic piezoviscous problem in Vázquez [15] and only to existence in several elastohydrodynamic isoviscous problems in Durany-Vázquez [9] and Bayada-Durany-Vázquez [3]. More recently, the existence of a solution has been proved for an elastohydrodynamic piezoviscous case with Dirichlet boundary condition in Bayada-El AlaouiVázquez [4]. The main objective of this work is the extension of this last result to the mixed Dirichlet-Neumann boundary condition. The need of this type of boundary condition is motivated by the transversal supply of fluid in the physical device treated here.

\section{The model problem}

In the book of Dowson-Higginson [8], a large variety of contacts in industrial devices are reduced to a ball-plane geometry by using the equivalent radius concept. So the elastohydrodynamical contact here considered consists of an elastic ball that rotates above a sliding rigid plane. They are separated by a thin film of lubricant supplied through an axial groove as shown in Fig. 1. The classical approximation of the gap is given in the rigid case by the expression

$$
h_{0}(x, y)=h^{0}+\frac{x^{2}+y^{2}}{2 R}
$$

that represents a parabolic approximation for a given sphere whose radius is $R$. The positive constant $h^{0}$ corresponds to the gap at the point nearest to contact (placed at the origin of coordinates). Clearly, the condition

$$
0<h^{0}<h_{0}(x, y)<h^{1} \text {, with } h^{0}, h^{1} \text { two constants }
$$

is satisfied in a bounded domain $\Omega$.

In the case of elastic surfaces an additional deformation term to expression (2.1) must be considered (see Oden-Wu [13]). Therefore, the total gap also depends on the fluid pressure in the following way:

$$
h=h(x, y, p)=h_{0}(x, y)+\frac{2}{\pi E} \int_{\Omega} \frac{p(t, u)}{\sqrt{(x-t)^{2}+(y-u)^{2}}} d t d u
$$



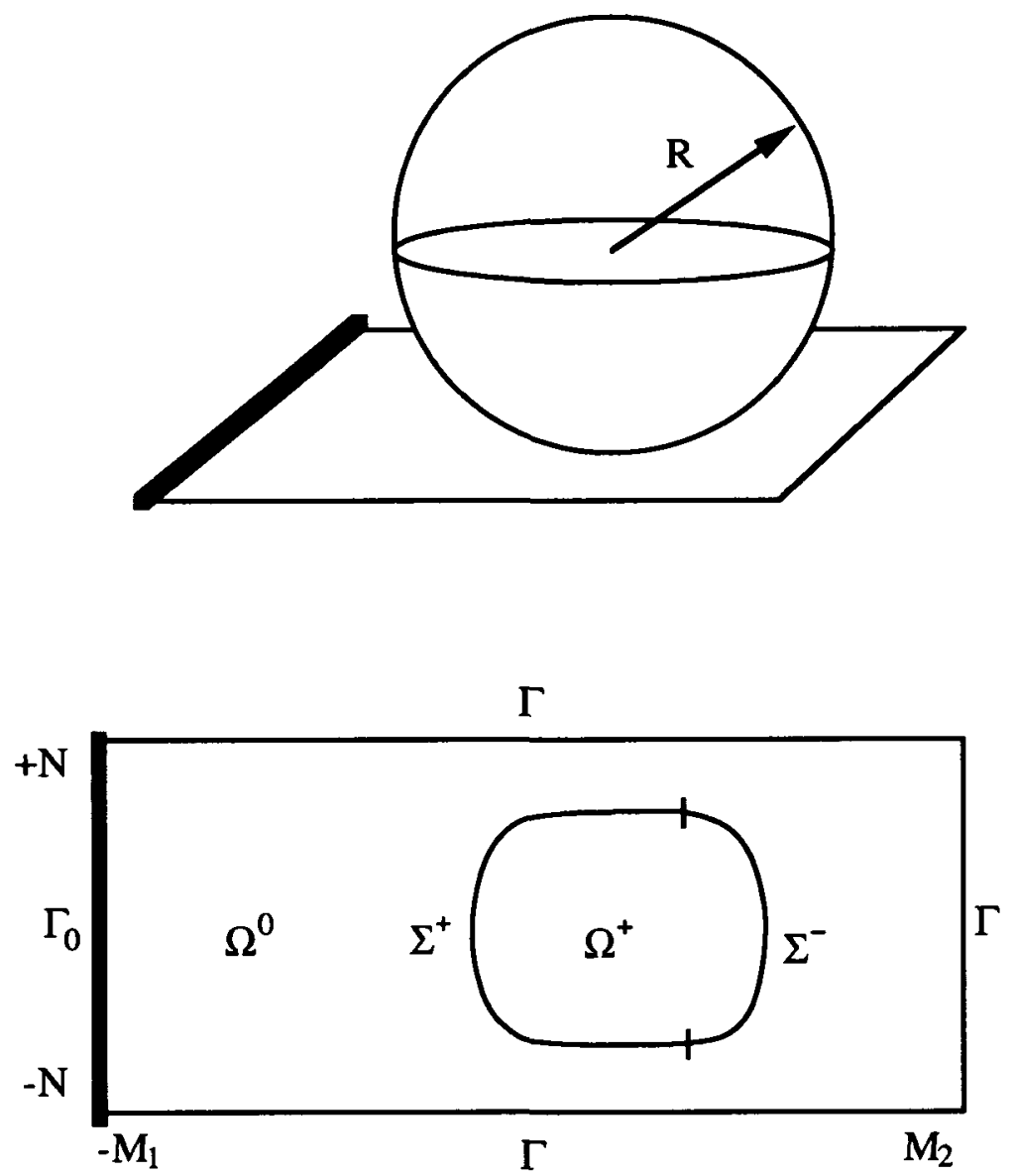

FIGURE 1

Lubricated device and bidimensional domain.

where $E$ represents the Young equivalent modulus. It can be proved that the function

$$
k((x, y),(t, u))=\frac{2}{\pi E \sqrt{(x-t)^{2}+(y-u)^{2}}}
$$

belongs to $L^{1}(\Omega)$ in the variable $(t, u)$ and the corresponding norm is bounded by a positive constant denoted hereafter by $K$.

The pressure-viscosity law of Barus for the homogeneous lubricant obeys the equation 


$$
v(p)=v_{0} \mathrm{e}^{\alpha p}
$$

where $\alpha$ and $v_{0}$ denote the piezoviscosity constant and the zero pressure viscosity respectively.

The whole mathematical strong formulation of the problem is posed on a bidimensional domain $\Omega=\left(-M_{1}, M_{2}\right) \times(-N, N)$ with $M_{1}, M_{2}$ and $N$ positive constants. Physically, it corresponds to a small region located in a neighbourhood of the nearcontact point in the rigid case. The set of equations modelling the coupled problem is:

Find $(p, \theta)$ such that:

$$
\begin{gathered}
\frac{\partial}{\partial x}\left(e^{-\alpha p} h^{3} \frac{\partial p}{\partial x}\right)+\frac{\partial}{\partial y}\left(e^{-\alpha p} h^{3} \frac{\partial p}{\partial y}\right)=6 v_{0} s \frac{\partial h}{\partial x}, \quad p>0 \text { and } \theta=1 \text { in } \Omega^{+} \\
\frac{\partial}{\partial x}(\theta h)=0, \quad p=0 \text { and } 0 \leqq \theta \leqq 1 \text { in } \Omega_{0} \\
h^{3} e^{-\alpha p} \frac{\partial p}{\partial n}=6 v_{0} s(1-\theta) h \cos (\vec{n}, \vec{i}), \quad p=0 \text { on } \Sigma
\end{gathered}
$$

with the boundary conditions

$$
\begin{gathered}
\theta=\theta_{0} \text { on } \Gamma_{\mathrm{o}} \\
p=0 \text { on } \Gamma
\end{gathered}
$$

where $s$ denotes the velocity of fluid displacement in the $x$ direction, $\theta_{0}$ is a supply parameter, $\vec{n}$ represents the unit normal vector to $\Sigma$ pointing to $\Omega_{0}, \vec{i}$ is the unit vector in the $x$-direction; and the sets appearing above are defined by

$$
\begin{aligned}
& \Omega^{+}=\{(x, y) \in \Omega / p(x, y)>0\} \\
& \Omega_{0}=\{(x, y) \in \Omega / p(x, y)=0\} \\
& \Sigma=\partial \Omega^{+} \cap \Omega \\
& \Gamma_{0}=\left\{(x, y) \in \partial \Omega / x=-M_{1}\right\} \\
& \Gamma=\partial \Omega \backslash \Gamma_{0} .
\end{aligned}
$$

In order to complete the above set of equations the gap-pressure relation (2.3) must be added.

Remark 2.1. The boundary conditions associated with the nonlinear elliptic partial differential equation correspond to a mixed Dirichlet-Neumann type problem. The Neumann condition is imposed on the supply boundary $\Gamma_{0}$ while on the rest of the 
boundary a homogeneous Dirichlet condition is considered. The equation (2.6) represents the Reynolds equation in the fluid part of the domain denoted by $\Omega^{+}$.

Let $H^{1}(\Omega)$ denote the classical Sobolev space and let $V$ be the space

$$
V=\left\{\phi \in H^{1}(\Omega), \phi=0 \text { on } \Gamma\right\}
$$

equipped with the norm

$$
\|\phi\|_{V}=\left(\int_{\Omega}|\nabla \phi|^{2} d x d y\right)^{1 / 2}
$$

that is equivalent to the norm usually considered in $H^{1}(\Omega)$.

We propose the following variational formulation:

Problem (P). For $\theta_{0} \in L^{2}\left(\Gamma_{0}\right), 0 \leqq \theta_{0} \leqq 1$, find $p \in H_{0}^{1}(\Omega)$ and $\theta \in L^{\infty}(\Omega)$ such that:

$$
\begin{gathered}
\int_{\Omega} h^{3}(p) \mathrm{e}^{-\alpha p} \nabla p \nabla \phi d x d y=6 v_{0} s \int_{\Omega} h(p) \theta \frac{\partial \phi}{\partial x} d x d y+6 v_{0} s \int_{\Gamma_{0}} h(p) \theta_{0} \phi d \sigma \quad \forall \phi \in V \\
H(p) \leqq \theta \leqq 1 \text { in } \Omega \\
p \geqq 0 \text { in } \Omega
\end{gathered}
$$

where $H$ denotes the Heaviside function defined by

$$
H(t)= \begin{cases}1 & t>0 \\ 0 & t \leqq 0\end{cases}
$$

and $h(p)$ is given by expression (2.3).

\section{Existence of solution}

In order to obtain an existence of solution theorem for problem $(\mathscr{P})$ we follow the classical method of regularization of the Heaviside map as in other related problems treated in Bayada-Chambat [2] and Vázquez [15]. This technique introduces the socalled regularized problem

Problem $\left(\mathscr{P}_{\imath}\right)$. Find $p_{\varepsilon} \in V$ such that

$$
\begin{gathered}
\int_{\Omega} h^{3}(p) e^{-\alpha} p_{\varepsilon} \nabla p_{\varepsilon} \nabla \phi d x d y=6 v_{0} s \int_{\Omega} H_{\varepsilon}\left(p_{\varepsilon}\right) h\left(p_{\varepsilon}\right) \frac{\partial \phi}{\partial x} d x d y \\
+6 v_{0} s \int_{\Gamma_{0}} \theta_{0} h\left(p_{\varepsilon}\right) \phi d \sigma \quad \forall \phi \in V
\end{gathered}
$$




$$
p_{\varepsilon} \geqq 0
$$

where the Heaviside function $H$ is approached by

$$
H_{\varepsilon}(t)=\left\{\begin{array}{rl}
1 & t>\varepsilon \\
t / \varepsilon & 0 \leqq t \leqq \varepsilon \\
0 & t \leqq 0
\end{array}\right.
$$

In order to demonstrate the existence of solution of the above regularized problem $\left(\mathscr{P}_{\varepsilon}\right)$ we propose some fixed-point theorem tools.

Let us define the subset $B_{R}$ of the Hilbert space $L^{2}(\Omega)$ as

$$
B_{R}=\left\{\phi \in L^{2}(\Omega) / 0 \leqq \phi \leqq R \text { a.e. in } \Omega\right\}
$$

and the operator

$$
\begin{aligned}
T: B_{R} & \rightarrow L^{2}(\Omega) \\
p & \rightarrow T(p)=q
\end{aligned}
$$

where $q$ is the solution of the following linear problem.

Problem (2). Find $q \in V$ such that

$$
\begin{gathered}
\int_{\boldsymbol{\Omega}} h^{3}(p) e^{-\alpha p} \nabla q \nabla \phi d x d y=6 v_{0} s \int_{\Omega} H_{\varepsilon}(p) h(p) \frac{\partial \phi}{\partial x} d x d y \\
+6 v_{0} s \int_{\Gamma_{0}} \theta_{0} h(p) \phi d \sigma \quad \forall \phi \in V
\end{gathered}
$$

where we have dropped the $\varepsilon$ index in the clearly $\varepsilon$-dependent solution.

Remark 3.1. For a given $p$ in $B_{R}$ the function $h(p)$ is well defined on $\Gamma_{0}$ in the sense of traces and belongs to $L^{2}\left(\Gamma_{0}\right)$ by means of the straightforward bound of the integrals involved. In fact, the function $h_{0}$ belongs to $L^{2}\left(\Gamma_{0}\right)$ and the inequality

$$
\left(\int_{-N}^{N}\left(\int_{\Omega} k\left(\left(-M_{1}, y\right),(t, u)\right) p(t, u) d t d u\right)^{2} d y\right)^{1 / 2} \leqq R L^{1 / 2} K
$$

holds with $L=2 N$, the length of $\Gamma_{0}$.

In order to study the linear problem (2) we proceed in three steps:

Step 1. Existence and $L^{2}(\Omega)$-estimates for problem (2). 
By the estimate

$$
\begin{aligned}
&\|h\|_{L^{\infty}(\Omega)} \leqq h^{1}+\left\|\int_{\Omega} k((x, y),(t, u)) p(t, u) d t d u\right\|_{L^{\infty}(\Omega)} \\
& \leqq h^{1}+R\left\|\int_{\Omega} k((x, y),(t, u)) d t d u\right\|_{L^{\infty}(\Omega)} \leqq h^{1}+K R
\end{aligned}
$$

the inequality

$$
0<\mathrm{e}^{-\alpha R} h^{0} \leqq \mathrm{e}^{-\alpha p} h \leqq h^{1}+K R
$$

leads to the existence and uniqueness of solution for the linear problem (2). Moreover we can take $\phi=q$ as a test function to have as $p$ lies in $B_{R}$

$$
\begin{aligned}
\left(h^{0}\right)^{3} \mathrm{e}^{-\alpha R} \int_{\Omega}|\nabla q|^{2} d x d y & \leqq 6 v_{0} s\left[\left(h^{1}+K R\right) \int_{\Omega}\left|\frac{\partial q}{\partial x}\right| d x d y+\|h\|_{L^{2}\left(\Gamma_{0}\right)}\|q\|_{L^{2}\left(\Gamma_{0}\right)}\right] \\
& \leqq 6 v_{0} s\left[\left(h^{1}+K R\right)|\Omega|^{1 / 2}+C(\Omega)\left(h^{1} L^{1 / 2}+R L^{1 / 2} K\right)\right]\|q\| V .
\end{aligned}
$$

Therefore

$$
\|\nabla q\|_{L^{2}(\Omega)} \leqq \frac{6 v_{0} s \mathrm{e}^{\alpha R}\left(h^{1}+K R\right)\left(|\Omega|^{1 / 2}+C(\Omega) L^{1 / 2}\right)}{\left(h^{0}\right)^{3}}
$$

with $C(\Omega)$ the norm of the trace mapping from $H^{1}(\Omega)$ into $L^{2}\left(\Gamma_{0}\right)$.

Step 2. The function $q \in H_{0}^{1}(\Omega) \cap C(\bar{\Omega})$.

For a real parameter $\lambda$ and a pair of given functions $f, g \in L^{\infty}(\Omega)$, such that $f>0$ and there exists a strictly positive constant $\mu$ satisfying $g \geqq \mu$, we consider the variational inequality:

Find $p_{r} \in K_{r}$ such that

$$
\int_{\mathbf{\Omega}} g \nabla p_{r} \nabla\left(\phi-p_{r}\right) d x d y \geqq \int_{\Omega} f \frac{\partial}{\partial x}\left(\phi-p_{r}\right) d x d y \quad \forall \phi \in K_{r}
$$

where

$$
K_{r}=\left\{\phi \in H_{0}^{1}(\Omega) / \phi \geqq 0\right\}
$$

Existence and uniqueness of solution for (3.9) are classical results of variational inequalities. Moreover, the following theorem holds. 
Theorem 3.1. Let $f \in L^{\infty}(\Omega) \cap L^{2}\left(\Gamma_{0}\right)$ be such that $f>0, g \in L^{\infty}(\Omega) \cap L^{2}\left(\Gamma_{0}\right)$ be such that there exists $\mu$ satisfying $g \geqq \mu>0$ in $\Omega$, and $\theta_{0} \in L^{2}\left(\Gamma_{0}\right)$ with $0 \leqq \theta_{0} \leqq 1$. Let $\bar{p}$ be the solution of the problem:

Find $\bar{p} \in V$ such that

$$
\int_{\mathbf{\Omega}} g \nabla \bar{p} \nabla \phi d x d y=\lambda \int_{\mathbf{\Omega}} H_{\varepsilon}(p) f \frac{\partial \phi}{\partial x} d x d y+\lambda \int_{\Gamma_{0}} \theta_{0} f \phi d \sigma \quad \forall \phi \in V
$$

If $p_{r}$ is the unique solution for (3.9) and if $\theta_{0}^{r}$ defined on $\Gamma_{0} b y$

$$
g \frac{\partial p_{r}}{\partial x}=\lambda\left(1-\theta_{0}^{r}\right) f
$$

satisfies $\theta_{0} \leqq \theta_{0}^{r}$ we have $\bar{p} \leqq p_{r}$.

Proof. The proof is a straightforward generalization of Theorem 3 in the work Durany-Vázquez [9] for the isoviscous case $(\alpha=0)$.

Corollary 3.1. Let $\theta_{0} \in L^{2}\left(\Gamma_{0}\right), 0 \leqq \theta_{0} \leqq 1$, and let $\theta_{0}^{r}$ be defined by (3.12). If $\theta_{0} \leqq \theta_{0}^{r}$, then the function $q$, the solution of $(3.4)$, belongs to $H_{0}^{1}(\Omega)$

Proof. Clearly, the choice of $g=h^{3}(p) \mathrm{e}^{-\alpha p}, f=h(p), \mu=\left(h^{0}\right)^{3} \mathrm{e}^{-\alpha R}$ and $\lambda=6 v_{0} s$ satisfies the hypotheses of the previous theorem.

Moreover the fact that $H_{\varepsilon}(p)$ and $h(p)$ belong to $H^{1}(\Omega)$ leads to the regularity property $q \in H^{2}(\Omega) \subset C(\bar{\Omega})$ and concludes this step.

Step 3. $L^{\infty}(\Omega)$-estimates for the linear problem.

After the previous computations we use the classical $L^{\infty}(\Omega)$ estimates for elliptic variational equations (see Kinderlehrer-Stampacchia [12] and Chipot [7], for example).

Thus, for a given $k \geqq 0$, Proposition 5.3 in [12], allows us to consider

$$
\xi_{k}=(q-k)^{+} \in H_{0}^{1}(\Omega) \subset V
$$

as a test function in (3.4). So, we have

$$
\begin{aligned}
\int_{\mathbf{\Omega}} h^{3}(p) e^{-a p} \nabla q \nabla \xi_{k} d x d y & =6 v_{0} s \int_{\mathbf{\Omega}} H_{\varepsilon}(p) h(p) \frac{\partial \xi_{k}}{\partial x} d x d y+6 v_{0} s \int_{\Gamma_{0}} \theta_{0} h(p) \xi_{k} d \sigma \\
& =6 v_{0} s \int_{\Omega \cap[q>k]} H_{\varepsilon}(p) h(p) \frac{\partial \xi_{k}}{\partial x} d x d y+6 v_{0} s \int_{\Gamma_{0} \cap \partial[q>k]} \theta_{0} h(p) \xi_{k} d \sigma .
\end{aligned}
$$


Nevertheless, the last integral on the boundary vanishes because $q \in H_{0}^{1}(\Omega) \cap C(\bar{\Omega})$. Therefore, the Hölder inequality leads to

$$
h^{0} \mathrm{e}^{-\alpha R} \int_{\Omega} h^{2}(p)\left|\nabla \xi_{k}\right|^{2} d x d y \leqq 6 v_{0} s\left(\int_{\Omega \cap[q>k]} h^{2}(p)\left|\frac{\partial \xi_{k}}{\partial x}\right|^{2} d x d y\right)^{1 / 2}|[q>k]|^{1 / 2}
$$

Thus

$$
\left[\int_{\Omega} h^{2}(p)\left|\nabla \xi_{k}\right|^{2} d x d y\right]^{1 / 2} \leqq \frac{6 v_{0} s \mathrm{e}^{\alpha R}}{h^{0}}|[q>k]|^{1 / 2}
$$

and

$$
\left\|\nabla \xi_{k}\right\|_{L^{2}(\Omega)} \leqq \frac{6 v_{0} s \mathrm{e}^{\alpha R}}{\left(h^{0}\right)^{2}}|[q>k]|^{1 / 2}
$$

For $k_{1} \geqq k_{2} \geqq 0$ we have $\left[q>k_{1}\right] \subset\left[q>k_{2}\right]$ and then

$$
\begin{aligned}
\left(k_{1}-k_{2}\right)^{p^{*}}\left|\left[q>k_{1}\right]\right| & =\int_{\left[q>k_{1}\right]}\left(k_{1}-k_{2}\right)^{p^{*}} d x d y \\
& \leqq \int_{\left[q>k_{1}\right]}\left|\xi_{k_{2}}\right|^{p^{*}} d x d y \leqq \int_{\left[q>k_{2}\right]}\left|\xi_{k_{2}}\right|^{p^{*}} d x d y \leqq C^{p^{*}}\left(\frac{6 v_{0} s \mathrm{e}^{\alpha R}}{\left(h^{0}\right)^{2}}\right)^{p^{*}}\left|\left[q>k_{2}\right]\right|^{p^{*} / 2}
\end{aligned}
$$

where the last inequality holds for $p^{*}>2$ by means of the continuity inclusion of $H_{0}^{1}(\Omega)$ into $L^{p^{*}}(\Omega)$ with the Sobolev constant $C$. Whence, we can state

$$
\left|\left[q>k_{1}\right]\right| \leqq\left(\frac{6 v_{0} s C \mathrm{e}^{\alpha R}}{\left(h^{0}\right)^{2}\left(k_{1}-k_{2}\right)}\right)^{p^{*}}\left|\left[q>k_{2}\right]\right|^{p^{*} / 2}
$$

and apply Lemma $B .1$. from Kinderlehrer-Stampacchia $[12$, p. 63] to establish the bound

$$
\|q\|_{L^{\infty}(\Omega)} \leqq \frac{6 v_{0} s \mathrm{e}^{\alpha R} C}{\left(h^{0}\right)^{2}}|\Omega|^{(1 / 2)-\left(1 / p^{*}\right)} 2^{\left(p^{*}\right) /\left(p^{*}-2\right)}
$$

for all $p^{*}>2$.

Now we can formulate the following proposition:

Proposition 3.1. For a given $p \in B$, there exists a solution of problem (2). Moreover we have the estimates:

$$
\|q\|_{v} \leqq \frac{6 v_{0} s e^{\alpha R}\left(h^{1}+K R\right)\left(|\Omega|^{1 / 2}+C(\Omega) L^{1 / 2}\right)}{\left(h^{0}\right)^{3}}=C_{1}
$$




$$
\|q\|_{L^{\infty}(\Omega)} \leqq \frac{6 v_{0} s e^{\alpha R} C}{\left(h^{0}\right)^{2}}|\Omega|^{(1 / 2)-\left(1 / p^{*}\right)} 2^{\left(p^{*}\right) /\left(p^{*}-2\right)}=C_{2}
$$

for all $p^{*}>2$.

The next technical lemma is useful for the required hypotheses for the application of the Schauder fixed point theorem.

Lemma 3.1. The functional from $L^{2}(\Omega)$ to $L^{2}(\Omega)$ defined by $p \in L^{2}(\Omega) \rightarrow h(p)$ is continuous.

Proof. See lemma 7.12 in Gilbarg-Trudinger ([10, p. 159]).

Theorem 3.2. If the Sobolev exponent $p^{*}>2$, the Sobolev constant $C$ and the physical parameters of the problem satisfy the condition:

$$
\frac{6 v_{0} s \alpha e C}{\left(h^{0}\right)^{2}}|\Omega|^{(1 / 2)-\left(1 / p^{*}\right)} 2^{\left(p^{*}\right) /\left(p^{*}-2\right)}<1
$$

then there exists a solution of the problem $\left(\mathscr{P}_{\varepsilon}\right)$ for every $\varepsilon>0$. Moreover we can obtain the $\varepsilon$-independent estimates:

$$
\begin{gathered}
\left\|p_{\varepsilon}\right\|_{H^{1}(\Omega)} \leqq C_{1}(1+C(\Omega)) \\
\left\|p_{\varepsilon}\right\|_{L^{\infty}(\Omega)} \leqq C_{2}
\end{gathered}
$$

Proof. The operator $T$ from $B_{R}$ to $L^{2}(\Omega)$ is compact and continuous. In fact, the continuity follows from Lemma 3.1 and the continuity of the exponential and $H_{\varepsilon}$ functions. Compactness is a direct consequence of the estimate (3.17) and the compact inclusion of $H^{1}(\Omega)$ into $L^{2}(\Omega)$.

In order to apply Schauder fixed point theorem we need the choice of a real positive number $R$ such that $T\left(B_{R}\right) \subset B_{R}$. It can easily be proved that $q$ is nonnegative by taking $q^{-}=\max (-q, 0)$ as a test function. So, it remains for $R$ to satisfy the inequality

$$
\frac{6 v_{0} s \mathrm{e}^{\alpha R} C}{\left(h^{0}\right)^{2}}|\Omega|^{(1 / 2)-\left(1 / p^{*}\right)} 2^{\left(p^{*}\right) /\left(p^{*}-2\right)} \leqq R
$$

But if condition (3.18) is satisfied then there exists an interval $\left(R_{0}, R_{1}\right)$ such that for all $R \in\left(R_{0}, R_{1}\right)$ the inclusion $T\left(B_{R}\right) \subset B_{R}$ holds. Let $f_{1}(R)$ and $f_{2}(R)$ be defined by

$$
\begin{gathered}
f_{1}(R)=\mathrm{e}^{\alpha R} \\
f_{2}(R)=A R,
\end{gathered}
$$


where

$$
A=\frac{\left(h^{0}\right)^{2}}{6 v_{0} s C|\Omega|^{(1 / 2)-\left(1 / p^{*}\right)} 2^{\left(p^{*}\right) /\left(p^{*}-2\right)}}
$$

Thus we need to establish the existence of $R$ such that $f_{1}(R) \leqq f_{2}(R)$. For this, it is enough for $A$ to be greater than the slope of the tangent line to $f_{1}$ that contains the origin. The translation of this geometrical argument leads to condition (3.18).

A solution of problem $(\mathscr{P})$ can be obtained as the limit of the sequence $\left\{p_{\varepsilon}\right\}_{\varepsilon}$ of regularized problems solutions.

Theorem 3.3. If condition (3.18) is fulfilled then there exists a solution of problem (P्P).

Proof. From (3.19) and (3.20) we have a subsequence of $\left\{p_{\varepsilon}\right\}_{\varepsilon}$, still denoted $\left\{p_{\varepsilon}\right\}_{\varepsilon}$, such that

$$
\exists p \in V \cap L^{\infty}(\Omega) / p_{\varepsilon} \rightarrow p \text { in } H^{1}(\Omega) \text { weakly and in } L^{\infty}(\Omega) \text { weakly-*. }
$$

Moreover

$$
\exists \theta \in L^{\infty}(\Omega) / H_{\varepsilon}\left(p_{\varepsilon}\right) \rightarrow \theta \quad \text { in } \quad L^{\infty}(\Omega) \text { weakly -*. }
$$

Therefore, from Theorem 3.2, the continuity of the exponential map and the previous convergences, we can obtain that the pair $(p, \theta)$ is a solution of the problem $(\mathscr{P})$ by passing to the limit in $\left(\mathscr{P}_{\varepsilon}\right)$.

\section{Conclusions}

In this paper the authors mainly generalize previous results of existence of solution for an isoviscous mathematical model to the more realistic piezoviscous case. Another original, but no less important, aspect is the supply of lubricant which is taken into account by means of a mixed Dirichlet-Neumann boundary condition.

Further effort is now being devoted to two research lines: the study of theoretical models that involve charge-imposed effects and the performance of numerical algorithms that allow approximated solutions to be obtained with a certain degree of accuracy.

Acknowledgements. This work has been partially supported by the Research Projects of D.G.I.C.Y.T. (PB92-0323) and Xunta de Galicia (XUGA21005B93).

\section{REFERENCES}

1. G. Bayada and $M$. Chambat Existence and uniqueness for a lubrication problem with nonregular conditons on the free boundary, Boll. Un. Mat. Ital. B 6 (3-B) (1984), 543-557.

2. G. Bayada and M. Chambat, Sur quelques modelisations de la zone de cavitation en lubrication hydrodynamique, J. Theor. Appl. Mech. 5 (1986), 703-729. 
3. G. Bayada, J. Durany and C. Vazquez, Existence of solutions for a lubrication problem in elastic journal bearing devices with thin bearing, Math. Methods Appl. Sci. 18 (1995), 255-266.

4. G. Bayada, M. El Alaoui and C. Vazquez, Existence of Solution for Elastohydrodynamic Piezoviscous Lubrication Problems with a New Model for Cavitation (Publication de l'Equipe d'Analyse Numérique Lyon-Saint Etienne, 163, 1994) and European J. Appl. Math. (1995), to appear.

5. H. Brezis, H. Kinderlehrer and G. Stampacchia, Sur une nouvelle formulation du probléme de l'écoulement á travers une digue, C. R. Acad. Sci. Paris Sér A-B (1978), 711-714.

6. A. CAmeron, Basic Lubrication Theory (Ellis Horwood Series, 1981).

7. M. Снipot, Variational Inequalities and Flow on Porous Media (Applied Math. Sciences Series 52, New York. Springer-Verlag, 1984).

8. D. Dowson and G. R. Higginson, Elastohydrodynamic Lubrication (Pergamon Press, 1977).

9. J. Durany and C. VÁzquez, Mathematical analysis of an elastohydrodynamic lubrication problem with cavitation, App. Anal. 53 (1994), 135-142.

10. D. Gilbarg and N. S. Trudinger, Elliptic Partial Differential Equations of Second Order (Berlin. Springer-Verlag, 1977).

11. B. Hu, A quasivariational inequality arising in elastohydrodynamics, SIAM J. Math. Anal. 21 (1990), 18-36.

12. D. Kinderlehrer and G. Stampacchia, An Introduction to Variational Inequalities and their Applications (New York. Academic Press, 1980).

13. J. T. ODEN and S. R. Wu, Existence of solutions to the Reynolds equation of elastohydrodynamic lubrication, Internat. J. Engrg. Sci. 23 (1985), 207-215.

14. J. F. Rodrigues, Remarks on the Reynolds problem of elastohydrodynamic lubrication, European J. Appl. Math. 4 (1993), 83-96.

15. C. VÃzQuez, Existence and uniqueness of solution for a lubrication problem with cavitation in a journal bearing with axial supply, Adv. in Math. Sci. and Appl. 4 (1994), 313-331.

Department of Applied Mathematics

UNIVERISTY OF VIGo

36280-VIGO

SPAIN 